\title{
Lasing without inversion in three-level systems without external coherent driving
}

\author{
V. Ahufinger, J. Mompart, and R. Corbalán* \\ Departament de Física, Universitat Autònoma de Barcelona, E-08193 Bellaterra, Barcelona, Spain
}

(Received 22 November 1999; published 17 April 2000)

\begin{abstract}
We have studied an incoherently pumped laser operating with a Doppler-broadened three-level system placed in a doubly resonant cavity. This system generates two laser fields, one of them without population inversion. Both ladder and V-type three-level schemes are considered with a ratio $R=\omega_{\alpha} / \omega_{\beta}$ of inversionless laser frequency $\omega_{\alpha}$ to ordinary laser frequency $\omega_{\beta}$ of $R=0.67$ and $R=1.88$, respectively. Dual-wavelength lasing extends up to Doppler-broadening values for optical transitions of atoms in a vapor cell. Some considerations for the practical realization of this dual-wavelength laser are discussed.
\end{abstract}

PACS number(s): 42.50.Gy, 42.40.Ht, 32.80.Qk

\section{INTRODUCTION}

The presence of atomic coherence manifests itself in a wealth of phenomena whose study is a topic of current interest in quantum optics and laser physics [1]. The essential feature of these phenomena is that the atomic coherence induced in a multilevel system by applying a strong "drive" laser field modifies the response of the medium to a weak probe field. Among the most interesting of these phenomena is lasing without inversion (LWI), because of its potential for short-wavelength lasing (e.g., in the UV or x-ray spectral domains) [2]. For conventional lasers of frequency $\omega$ based on population inversion, continuous wave (cw) lasing in these spectral domains is unpractical because the required incoherent pump power scales approximately as $\omega^{4}$ for Dopper-broadened transitions or as $\omega^{6}$ for natural broadening [3] due to the $\omega^{3}$ scaling of the Einstein $A$ coefficient. In LWI a coherent driving field acting on one transition generates the atomic coherence that relaxes the population inversion condition for laser oscillation in an adjacent transition. This greatly reduces the minimum excited state population required for lasing. In previous theoretical work, as well as in the three recent experimental demonstrations of LWI in atomic vapors [4-6], the coherence-generating drive field was imposed from the outside. In these proofs of principle experiments the frequency upconversion ratio $R \equiv \omega_{\alpha} / \omega_{\beta}$ (i.e., the ratio of generated laser frequency $\omega_{\alpha}$ to drive laser frequency $\omega_{\beta}$ ) was either smaller or very close to 1 . This was dictated by the desire to mitigate or avoid the negative influence of Doppler broadening for LWI [7].

Here we consider a Doppler-broadened three-level system placed in a doubly resonant cavity (i.e., the cavity is resonant with both dipole allowed atomic transitions; see Fig. 1) and driven only by external incoherent pump mechanisms [not shown in Fig. 1(a)]. We look for conditions such that each dipole-allowed transition generates a laser field, one of them (denoted by $\alpha$ ) without population inversion while the other (the drive, denoted by $\beta$ ) with population inversion. This system should be advantageous, especially in the frequency upconversion regime $(R>1)$, due to the high drive field

*FAX: +34 935812155 .

Electronic address: Ramon.Corbalan@uab.es strength available, since the inversionless laser cavity is integrated into the drive laser resonator. In fact, it is well known that Doppler broadening is one of the main difficulties for LWI when the frequencies of the laser and drive fields are substantially different $[7,8]$ and that a possible way to mitigate this problem is to use a strong drive field such that the associated Rabi frequency is on the order of the Doppler width [7]. If the drive field is imposed from the

(a)

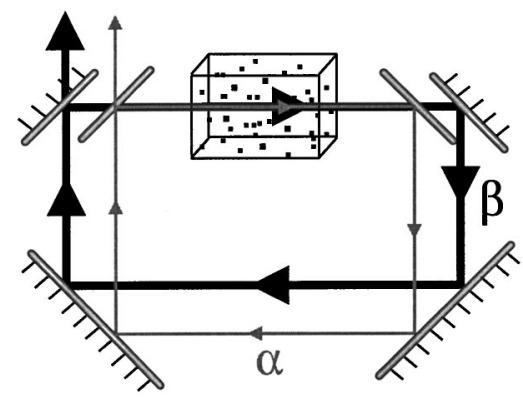

(b)

(c)
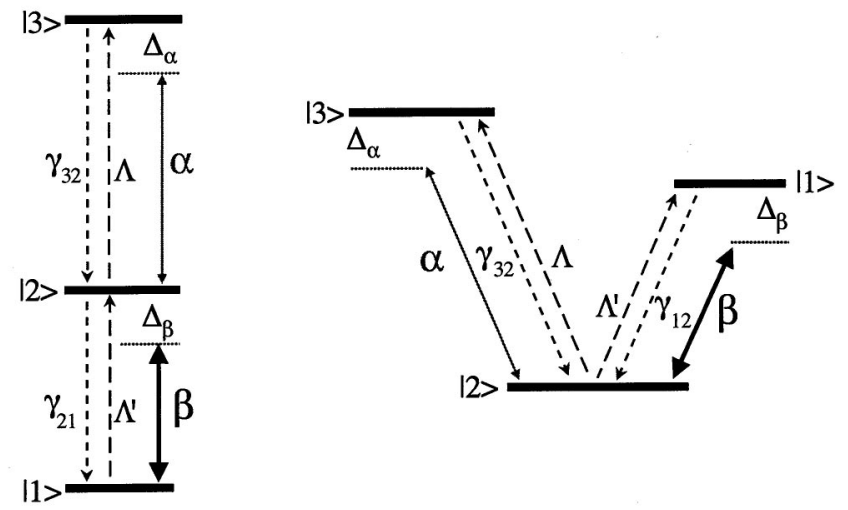

FIG. 1. (a) Dual-wavelength laser under investigation. The material medium is considered either as a three-level ladder system or as a V system. (b) Ladder scheme. (c) V scheme. $\alpha$ and $\beta$ ( $\Delta_{\alpha}$ and $\Delta_{\beta}$ ) are half the Rabi frequencies (the detunings from atomic resonance) of the two generated laser fields. $\gamma_{12}, \gamma_{21}$, and $\gamma_{32}$ are spontaneous population decay rates. $\Lambda$ and $\Lambda^{\prime}$ are population transfer rates associated with two incoherent pumping processes. 
outside, such high intensities would be normally available only in the form of short pulses. Instead we here study our dual-wavelength laser in the $\mathrm{cw}$ regime.

Our system is also interesting from at least another perspective: While in ordinary LWI systems the drive field intensity and detuning are control parameters, here they are dynamical variables and the nonlinear interaction between drive and laser fields through the atomic medium could result in interesting dynamic behavior. However, we mainly restrict ourselves here to study the steady state of our laser system and leave its dynamics for future work.

\section{MODEL AND RESULTS}

Consider a three-level system of the ladder type or the $\mathrm{V}$ type [see Figs. 1(b) and 1(c), respectively] excited by two incoherent pump mechanisms characterized by unidirectional rates $\Lambda$ and $\Lambda^{\prime}$. The pump rate $\Lambda^{\prime}$ is considered large enough to invert the population at the transition $|2\rangle \leftrightarrow|1\rangle$. On the contrary, the other incoherent pump does not invert the population in the transition $|3\rangle \leftrightarrow|2\rangle$. The three-level system is placed in a doubly resonant cavity [Fig. 1(a)] and can therefore generate a laser field from the transition $|2\rangle \leftrightarrow|1\rangle$, characterized by a Rabi frequency $2 \beta$ and a detunig $\Delta_{\beta}$ from atomic resonance. Hereafter we call this field the drive field. It generates the atomic coherence that can relax the population inversion condition for laser oscillation in the adjacent transition. The Rabi frequency $2 \alpha$ and the detuning $\Delta_{\alpha}$ characterize the coupling of the inversionless laser field with the atomic medium.

\section{A. Ladder system}

To be specific let us consider the Doppler-broadened ladder or cascade scheme of Fig. 1(b). Using standard semiclassical methods and the rotating wave, uniform field, and slowly varying envelope approximations, we find the following set of Maxwell-Schrödinger equations of motion for the atomic density matrix elements $\rho_{i j}(i, j=1,2,3)$ and Rabi frequencies $2 \alpha$ and $2 \beta$ :

$$
\begin{gathered}
\dot{\rho}_{11}=\gamma_{21} \rho_{22}-\Lambda^{\prime} \rho_{11}+2 \beta y_{12} \\
\dot{\rho}_{22}=-\gamma_{21} \rho_{22}+\Lambda^{\prime} \rho_{11}-\Lambda \rho_{22}+\gamma_{32} \rho_{33}-2 \beta y_{12}+2 \alpha y_{23}, \\
\dot{\rho}_{33}=-\gamma_{32} \rho_{33}+\Lambda \rho_{22}-2 \alpha y_{23}, \\
\dot{\rho}_{12}=-\left[\Gamma_{12}+i \Delta_{\beta}(v)\right] \rho_{12}-i \alpha \rho_{13}+i \beta\left(\rho_{22}-\rho_{11}\right) \\
\dot{\rho}_{13}=-\left\{\Gamma_{23}+i \Delta_{\alpha}(v)\right] \rho_{23}+i \beta \rho_{13}+i \alpha\left(\rho_{33}-\rho_{22}\right),
\end{gathered}
$$$$
\dot{\alpha}=-\kappa_{\alpha} \alpha+\int g_{23}(v) y_{23}(v) d v,
$$

$$
\dot{\beta}=-\kappa_{\beta} \beta+\int g_{12}(v) y_{12}(v) d v
$$

where $\rho_{i j} \equiv x_{i j}+i y_{i j}$ is the coherence between states $|i\rangle$ and $|j\rangle$ and $\rho_{i i}$ is the population of level $|i\rangle . \gamma_{21}$ and $\gamma_{32}$ are the population decay rates indicated in Fig. $1(\mathrm{~b}) . \Gamma_{i j}$ is the coherence decay rate; in the so-called radiative limit, when there are no dephasing processes, one has $\Gamma_{13}=\left(\gamma_{32}\right.$ $\left.+\Lambda^{\prime}\right) / 2, \quad \Gamma_{23}=\left(\gamma_{21}+\gamma_{32}+\Lambda\right) / 2, \quad$ and $\quad \Gamma_{12}=\left(\gamma_{21}+\Lambda\right.$ $\left.+\Lambda^{\prime}\right) / 2$. The velocity-dependent detunings $\Delta_{\alpha}(v)$ and $\Delta_{\beta}(v)$ are given by

$$
\begin{aligned}
& \Delta_{\alpha}(v)=\Delta_{\alpha}+s \omega_{\alpha} \frac{v}{c}, \\
& \Delta_{\beta}(v)=\Delta_{\beta}+\omega_{\beta} \frac{v}{c},
\end{aligned}
$$

where $\Delta_{\alpha}$ and $\Delta_{\beta}$ are the nominal detunings, $v$ the atomic velocity toward the drive laser beam, $c$ the velocity of light, and $s$ a parameter that indicates whether the two fields are co- or counterpropagating ( $s=1$ or $s=-1$, respectively). $\kappa_{\beta}\left(\kappa_{\alpha}\right)$ is the damping rate of the drive (inversionless) laser due to cavity losses and $g_{12}(v)=N(v) \omega_{21} \mu_{12}^{2} / 2 \hbar \varepsilon_{0}$ and $g_{23}(v)=N(v) \omega_{32} \mu_{23}^{2} / 2 \hbar \varepsilon_{0}$ the gain parameters for the corresponding lasing transition, where $\omega_{21}\left(\omega_{32}\right)$ is the transition frequency, $\mu_{12}\left(\mu_{23}\right)$ the electric dipole matrix element, $N(v) d v$ is the density of atoms with velocity $v, \hbar$ Planck's constant, and $\varepsilon_{0}$ the dielectric permittivity. Notice that in a medium with a purely radiative decay as is assumed here one has $g_{12} / g_{23} \approx R^{2} \gamma_{21} / \gamma_{32}$ where $R=\omega_{\alpha} / \omega_{\beta} \approx \omega_{32} / \omega_{21}$ is the frequency upconversion ratio. Finally, the detunings $\Delta_{\alpha}$ and $\Delta_{\beta}$ are linked to the cavity detunings $\Delta_{\alpha}^{c}$ and $\Delta_{\beta}^{c}$ from the respective atomic resonance $\left(\omega_{32}\right.$ and $\left.\omega_{21}\right)$ by the relations

$$
\begin{aligned}
& \Delta_{\alpha}=\Delta_{\alpha}^{c}-\int g_{23}(v) \frac{x_{23}(v)}{\alpha} d v, \\
& \Delta_{\beta}=\Delta_{\beta}^{c}-\int g_{12}(v) \frac{x_{12}(v)}{\beta} d v .
\end{aligned}
$$

Therefore, $\Delta_{\alpha}^{c}$ and $\Delta_{\beta}^{c}$ are constant control parameters but not $\Delta_{\alpha}$ and $\Delta_{\beta}$ which are dependent dynamical variables since they are linked, respectively, to $x_{23}$ and $\alpha$ and to $x_{12}$ and $\beta$. For $N(v)$ we will take the standard Maxwellian distribution

$$
N(v)=\frac{N_{0}}{\sigma \sqrt{\pi}} \exp \left(-v^{2} / \sigma^{2}\right),
$$

where $\sigma$ is the most probable velocity. To characterize the velocity distribution we will use the well-known expression of the full width at half maximum of the resonance line of a purely Doppler-broadened vapor, given for the inversionless lasing transition by

$$
\Delta \omega_{D \alpha}=2 \sqrt{\ln 2} \frac{\sigma}{c} \omega_{\alpha}
$$


In the following, for the sake of definiteness, we will use parameters of a real cascade system in atomic ${ }^{138} \mathrm{Ba}$ with $\lambda_{32}=821 \mathrm{~nm}$, and $\lambda_{21}=554 \mathrm{~nm}$, i.e., $R=0.67, \gamma_{32}=3.5 \mathrm{MHz}$ and $\gamma_{21}=19 \mathrm{MHz}$ [9], which has already been used experimentally by Sellin et al. [10]. Other parameters are fixed at the following values: $\Lambda=3.3 \mathrm{MHz}, \Lambda^{\prime}=25 \mathrm{MHz}, k_{\alpha}=1$ $\mathrm{MHz}, k_{\beta}=5 \mathrm{MHz}, g_{23}=1 \times 10^{5} \mathrm{MHz}^{2}$, and $g_{12}=2.5$ $\times 10^{5} \mathrm{MHz}^{2}$.

\section{Homogeneous broadening}

For simplicity, let us consider first the case in which Doppler broadening can be neglected. Mathematically, this case is obtained in the limit $\sigma \rightarrow 0$, in which the velocity distribution $N(v)$ behaves as a Dirac $\delta$ function: $N(v) \rightarrow N_{0} \delta(0)$. This means that all the atoms "see" both fields with their respective nominal detuning $\Delta_{\alpha}$ and $\Delta_{\beta}$ and, therefore, all the atoms respond to the fields in exactly the same way. The unsaturated gain parameters to be used now are the ones defined above with $N(v)$ replaced by $N_{0}$.

Since a coherent drive field is required to generate the field $\alpha$ without inversion, we first study the threshold condition for lasing at the $|1\rangle \leftrightarrow|2\rangle$ transition. Taking $\alpha$ and all time derivatives in Eqs. (1) equal to zero, and solving Eqs. (1a) $-(1 \mathrm{c})$ to zeroth order in the field $\beta$, and Eq. (1d) to first order in $\beta$, the condition $\kappa_{\beta}<g_{12} y_{12} / \beta$ [see Eq. (1h)] reads

$$
\kappa_{\beta}\left(\Delta_{\beta}^{2}+\Gamma_{12}^{2}\right)-g_{12} \Gamma_{12} n_{21}<0,
$$

where $n_{21} \equiv \rho_{22}-\rho_{11}$. In the limit $\kappa_{\beta} / g_{12} \rightarrow 0$ this equation simply states that lasing of the field $\beta$ occurs when $n_{21}>0$, i.e., when there is population inversion at the $|1\rangle \leftrightarrow|2\rangle$ transition, and this requires $\Lambda^{\prime}>\gamma_{21}$.

When condition (6) is fulfilled the drive field is generated. The steady-state value of its amplitude $\beta$ and detuning $\Delta_{\beta}$ are obtained by solving Eqs. (1) to all orders in $\beta$, with $\alpha$ and all the time derivatives equal to zero. Finally, solving Eqs. (1a)-(1d) to zeroth order and Eqs. (1e)-(1f) to first order of the field $\alpha$, we obtain the threshold condition for continuous wave generation of field $\alpha\left(\kappa_{\alpha}<g_{23} y_{23} / \alpha\right)$ which reads

$$
\begin{aligned}
c \equiv & \kappa_{\alpha}\left\{\beta^{2}\left[\beta^{2}-2 \Delta_{\alpha}\left(\Delta_{\alpha}+\Delta_{\beta}\right)+2 \Gamma_{13} \Gamma_{23}\right]+\Delta_{\alpha}^{2}\left[\left(\Delta_{\alpha}+\Delta_{\beta}\right)^{2}\right.\right. \\
& \left.\left.+\Gamma_{13}^{2}\right]+\left[\left(\Delta_{\alpha}+\Delta_{\beta}\right)^{2}+\Gamma_{13}^{2}\right] \Gamma_{23}^{2}\right\}-g_{23}\left\{n _ { 3 2 } \left[\beta^{2} \Gamma_{13}\right.\right. \\
& \left.+\Gamma_{23}\left(\Delta_{\alpha}+\Delta_{\beta}\right)^{2}+\Gamma_{13}^{2} \Gamma_{23}\right]-\beta x_{12}\left[\Delta_{\alpha} \Gamma_{13}+\left(\Delta_{\beta}\right.\right. \\
& \left.\left.\left.+\Delta_{\alpha}\right) \Gamma_{23}\right]+\beta y_{12}\left[\beta^{2}+\Gamma_{13} \Gamma_{23}-\Delta_{\alpha}\left(\Delta_{\alpha}+\Delta_{\beta}\right)\right]\right\}<0,
\end{aligned}
$$

where $n_{32}=\rho_{33}-\rho_{22}, x_{12}, y_{12}$, and $\beta$ and $\Delta_{\beta}$ correspond to the solution to zeroth order in $\alpha$. Figure 2(a) shows the curve $c=0$ as a function of $\Delta_{\alpha}$ and $\Delta_{\beta}$ (or $\Delta_{\beta}^{c}$ ). By comparing the scales of the two horizontal axis at the bottom in Fig. 2 one realizes that for the $\beta$ field there is an appreciable frequency pulling [described by Eq. (3b)] toward atomic resonance $\omega_{21}$. There is positive net gain $(c<0)$ for detunings corresponding to points inside the domain limited by the curve $c=0$. As is clearly seen the $\alpha$-lasing domain extends close to

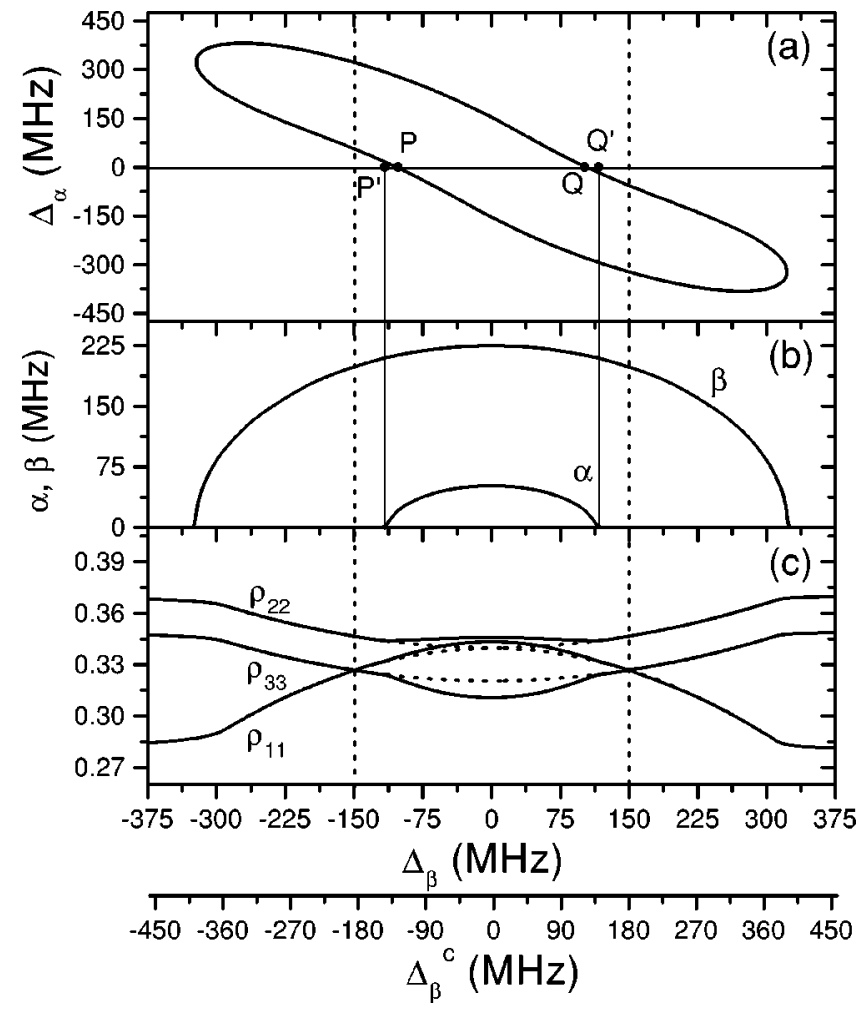

FIG. 2. (a) The area inside the curve corresponds to the LWI domain for the $\alpha$ field in the plane $\left(\Delta_{\alpha}, \Delta_{\beta}\right)$. (b) Amplitude of the generated fields versus $\Delta_{\beta}$, with $\Delta_{\alpha}^{c}=0$. (c) Atomic populations for parameters as in (b). Solid (dashed) lines correspond to the case in which both fields (only the $\beta$ field) are generated. Notice that twophoton inversion is absent only inside the domain limited by vertical dotted lines. Since $\Delta_{\beta}$ is not a control parameter, we have plotted also another scale with the corresponding values of the cavity detuning $\Delta_{\beta}^{c}$. For other parameters see text.

the diagonal $\Delta_{\alpha}=-\Delta_{\beta}$, i.e., close to the two-photon resonance condition. This was expected, since it is well known that two-photon processes are responsible for inversionless gain in cascade schemes with population inversion at the driven transition $[11,12]$.

Steady-state solutions of the Maxwell-Schrödinger equations (1), obtained numerically, are reported in Figs. 2(b) and 2(c). Figure 2(b) shows the amplitudes of the generated fields as a function of $\Delta_{\beta}$ (or $\Delta_{\beta}^{c}$ ), with $\Delta_{\alpha}^{c}=0$. The corresponding atomic populations are plotted in Fig. 2(c). Here solid (dashed) lines correspond to the case in which both fields (only the $\beta$ field) are generated. It is clearly seen that $\beta$ lasing occurs with population inversion $\left(\rho_{22}>\rho_{11}\right)$ while $\alpha$ lasing, in the domain limited by vertical dotted lines, takes place with population inversion neither at the one-photon transition $\left(\rho_{22}>\rho_{33}\right)$ nor at the two-photon transition $\left(\rho_{11}\right.$ $\left.>\rho_{33}\right)$. Outside this domain there is two-photon inversion $\left(\rho_{33}>\rho_{11}\right)$. The small cavity losses and the high gain at the transition $|2\rangle \leftrightarrow|1\rangle$, associated with population inversion, allow the generation of the $\beta$ field over a broad detuning range $\left(\left|\Delta_{\beta}\right|<324 \mathrm{MHz}=17 \gamma_{21}\right)$ with a large amplitude on resonance. Notice that in Fig. 2(a) the $\alpha$-lasing domain extends up to $\Delta_{\beta}$ values for which there is emission of the $\beta$ (drive) field [see Fig. 2(b)]. This is not the case in the latter figure 

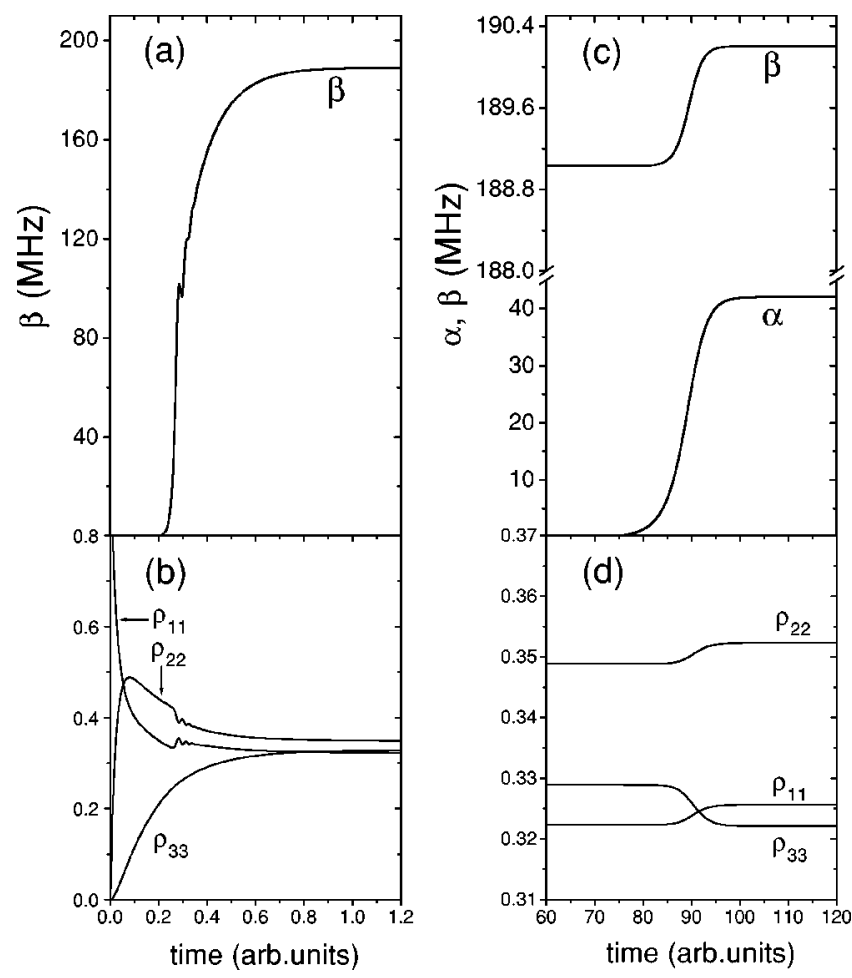

FIG. 3. Time evolution toward the steady state of the generated fields, (a) and (c), and the atomic level populations, (b) and (d). The two laser beams are counterpropagating, $\Delta_{\alpha}^{c}=\Delta_{\beta}^{c}=0, \Delta \omega_{D \alpha}$ $=366.3 \mathrm{MHz}$, and other parameters are given in the text.

because here $\Delta_{\alpha}^{c}$ was fixed to $\Delta_{\alpha}^{c}=0$. For an ordinary twolevel laser tuned to resonance there are no dispersive effects. If this were the case for the $\alpha$ laser, its lasing domain in Fig. 2(b) should extend only to $\Delta_{\beta}$ values corresponding to the segment $\overline{P Q}$ displayed in Fig. 2(a). Instead, the lasing domain is slightly enlarged covering detunings $\Delta_{\beta}$ in the segment $\overline{P^{\prime} Q^{\prime}}$. This is due to the presence of a detuned drive field which entails a frequency pushing for the $\alpha$ field. Notice that this results in $\Delta_{\alpha}$ shifting from resonance whose sign is opposite to that of $\Delta_{\beta}$. We have also observed that the modulus of the $\Delta_{\alpha}$ shift from resonance corresponding to the various points in the domain of lasing in Fig. 2(b) increases monotonically with $\left|\Delta_{\beta}\right|$. Thus, the frequency pushing tends to restore the two-photon resonance condition.

\section{Doppler broadening}

Figure 3 shows results of a numerical integration of Eqs. (1) with counterpropagating $\alpha$ and $\beta$ fields, $\Delta_{\alpha}^{c}=\Delta_{\beta}^{c}=0$, $\Delta \omega_{D \alpha}=366.3 \mathrm{MHz}$, and other parameters as specified above. The two incoherent pump mechanisms are switched on to their constant values at $t=0$. As seen in Figs. 3(a) and 3(b), a buildup of the $\beta$-laser oscillation starts soon after population inversion at the transition $|2\rangle-|1\rangle$ has been created. This field, together with the incoherent pump mechanisms, produces a population inversion at the two-photon transition $|3\rangle-|1\rangle$ [see Figs. 3(b) and 3(d)] and allows the generation of the $\alpha$ field with a delay that depends slightly on the initial $\alpha_{0}$ value taken for the integration. The $\beta$ laser

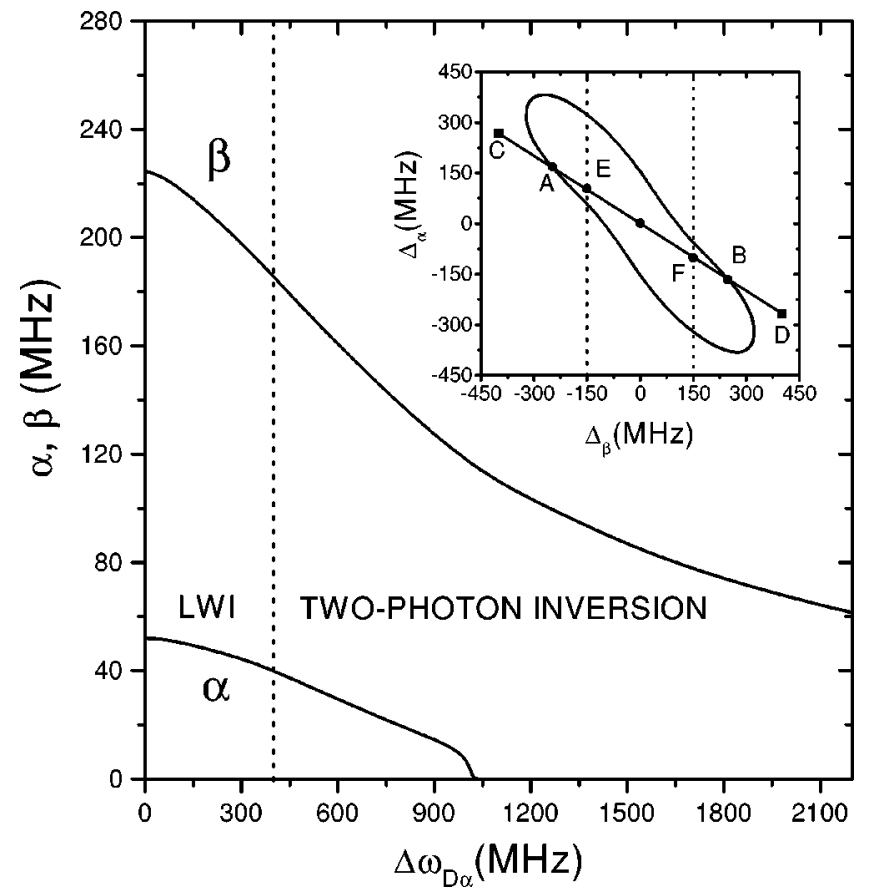

FIG. 4. Amplitude of the generated laser fields as a function of the Doppler broadening for $\Delta_{\alpha}^{c}=\Delta_{\beta}^{c}=0$. Other parameters are given in the text. The two laser beams are counterpropagating. True LWI of the $\alpha$ field takes place only at the left hand side of the vertical dotted line. At the right hand side there is population inversion for the two-photon transition. The inset reproduces the lasing domain of Fig. 2(a), and the points of the line $\overline{C D}$ represent different atomic velocity classes for $\Delta \omega_{D_{\alpha}}=210.4 \mathrm{MHz}$.

always operates with population inversion $\left(\rho_{22}>\rho_{11}\right)$. On the contrary, the transient two-photon inversion $\left(\rho_{33}>\rho_{11}\right)$ disappears in the steady state [see Fig. 3(d)] and one has true LWI for the $\alpha$ field $\left(\rho_{22}>\rho_{11}>\rho_{33}\right.$, i.e., population inversion neither at the one-photon transition nor at the twophoton transition). In this configuration the two fields cooperate: the $\beta$ field enables the inversionless generation of the $\alpha$ field and this, in its turn, produces an increase of the $\beta$ intensity [see Fig. 3(c)] which confirms the fact that $\alpha$-LWI is due to two-photon gain processes even without twophoton inversion.

The negative influence of Doppler broadening in our system can be appreciated in Fig. 4 where we plot the generated field amplitudes as a function of the Doppler broadening $\Delta \omega_{D \alpha}$, for the fully resonant case $\Delta_{\alpha}^{c}=\Delta_{\beta}^{c}=0$. The intensities of both fields decrease as $\Delta \omega_{D \alpha}$ increases and eventually $\alpha$ lasing disappears for rather large $\Delta \omega_{D \alpha}$ values $\left(\Delta \omega_{D \alpha}\right.$ $\sim 1 \mathrm{GHz}$ ). This is easily interpreted with the help of the inset of Fig. 4, which reproduces the $\alpha$-lasing domain of Fig. 2(a). Taking into account Eqs. (2) it results that the points representing different velocity classes on the lasing domain lie on a straight line passing through the origin with a slope $-R$ $=-0.67$. The length of this line depends on the width of the velocity distribution. In the inset we have considered the case $\Delta \omega_{D \alpha}=210.4 \mathrm{MHz}$ and plotted a line that covers the domain of detunings corresponding to three standard deviations in the velocity distribution. Since one has to consider 
the contribution of all the atoms simultaneously, it is clear that $\alpha$ lasing is only possible when the contribution of the amplifying atoms (those in the segment $\overline{A B}$ ) overcomes that of the absorbing atoms (those in the segments $\overline{A C}$ and $\overline{B D}$ ). Notice also that there is no population inversion at the twophoton transition only for atoms in the segment $\overline{E F}$. Therefore, when the Doppler broadening (i.e., the length of the segment $\overline{C D}$ ) increases, the velocity-integrated population in level $|3\rangle$ becomes larger than in the ground state $|1\rangle$. This is the case at the right hand side of the vertical dotted line in Fig. 4.

\section{B. V-type system}

We consider now that the material medium of the dualwavelength laser can be represented by the V-type threelevel system shown in Fig. 1(c). The Maxwell-Schrödinger equations for this laser can be obtained by changing in Eqs. (1) the terms corresponding to the incoherent interactions in a straightforward manner and $\Delta_{\beta}$ by $-\Delta_{\beta}$, so we do not write them here. We will analyze these equations for parameters appropriate for a $\mathrm{V}$ scheme in ${ }^{85} \mathrm{Rb}$ involving the transitions $5^{2} S_{1 / 2}-5^{2} P_{1 / 2}$ at $\lambda_{\beta}=794.8 \mathrm{~nm}$ and $5^{2} S_{1 / 2}-6^{2} P_{1 / 2}$ at $\lambda_{\alpha}=421.6 \mathrm{~nm}$, i.e., $R=1.88$. Other parameters are $\gamma_{12}$ $=6.37 \mathrm{MHz}, \gamma_{32}=1.43 \mathrm{MHz}, \Lambda=1.4 \mathrm{MHz}, \Lambda^{\prime}=10 \mathrm{MHz}$, $\kappa_{\alpha}=1 \mathrm{MHz}, \kappa_{\beta}=10 \mathrm{MHz}, g_{23}=0.67 \times 10^{5} \mathrm{MHz}^{2}$, and $g_{12}$ $=1 \times 10^{6} \mathrm{MHz}^{2}$.

\section{Homogeneous broadening}

Neglecting Doppler broadening and following the same procedure described in Sec. II A 1 we determine the generated $\beta$ field amplitude [Fig. 5(b)] as a function of $\Delta_{\beta}\left(\right.$ or $\left.\Delta_{\beta}^{c}\right)$ and the $\alpha$-lasing domain [Fig. 5(a)] that is now split into two subdomains symmetrically located about the origin $\Delta_{\alpha}=\Delta_{\beta}$ $=0$. Figure 5(c) shows that drive field emission takes place with population inversion at the one-photon transition $\left(\rho_{11}\right.$ $>\rho_{22}$ ) while $\alpha$ lasing occurs without population inversion either at the one-photon transition $\left(\rho_{33}<\rho_{22}\right)$ or at the Raman $|3\rangle \leftrightarrow|1\rangle$ two-photon transition $\left(\rho_{33}<\rho_{11}\right)$. Notice in Fig. 5(a) that for resonant driving there are two regions of $\alpha$ lasing symmetrically located about line center. This is a well-known feature of $\mathrm{V}$ schemes with population inversion at the driven transition [11-13]. It is also known that inversionless amplification of the $\alpha$ field in these systems is due to stimulated one-photon processes $[11,12]$. This fact qualitatively explains the shape of the domains in Fig. 5(a): At $\Delta_{\beta}=0$ the drive field $\beta$ is the strongest and splits symmetrically level $|2\rangle$ into two dressed states separated by $2 \beta$; amplification of the $\alpha$ field occurs at any of the two resonances involving the upper level $|3\rangle$ and these two lower dressed states, and therefore takes place at $\Delta_{\alpha}= \pm \beta$. At large enough $\Delta_{\beta}$ there is $\alpha$ gain only in the resonance involving level $|3\rangle$ and level $|2\rangle$ light shifted by $\beta^{2} / \Delta_{\beta}$. Therefore, $\alpha$ amplification takes place for a $\Delta_{\alpha}$ with a different sign from that of $\Delta_{\beta}$; moreover, since as $\left|\Delta_{\beta}\right|$ increases $\beta$ and the light shift decrease, the $\Delta_{\alpha}$ values at which $\alpha$ lasing occurs approach $\Delta_{\alpha}=0$.

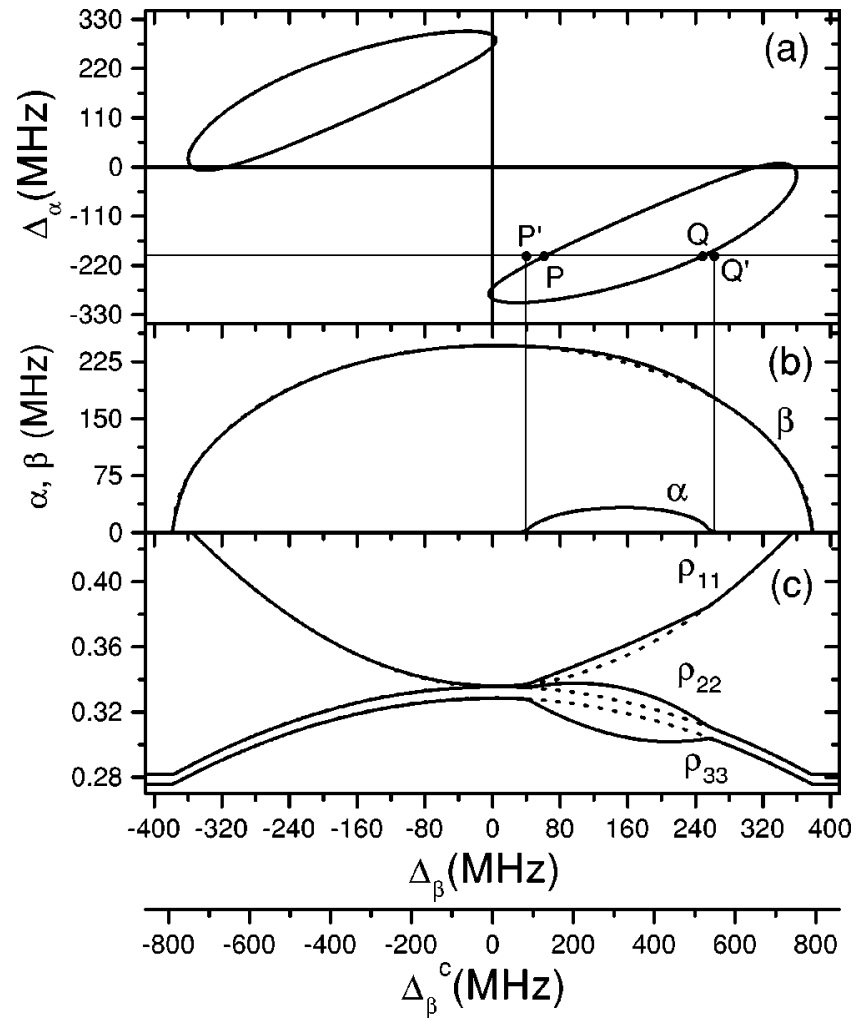

FIG. 5. The same as in Fig. 2 but now for the V scheme. In (b) $\Delta_{\alpha}^{c}=-200 \mathrm{MHz}$. Other parameters are given in the text.

A comparison of the two horizontal scales at the bottom of Fig. 5 shows that there is a strong frequency pulling of the drive field toward atomic resonance. Finally, Fig. 5(b) shows the generated $\alpha$ field as a function of $\Delta_{\beta}$ (or $\Delta_{\beta}^{c}$ ) with a cavity tuning fixed at $\Delta_{\alpha}^{c}=-200 \mathrm{MHz} . \alpha$ lasing occurs for $\Delta_{\beta}$ detunings corresponding to the segment $\overline{P^{\prime} Q^{\prime}}$ and not to the segment $\overline{P Q}$ as it would be the case in the absence of dispersive effects. We have observed that these effects are more pronounced near point $P$, resulting in frequency pushing for the $\alpha$ field, while there is frequency pulling near point $Q$.

\section{Doppler broadening}

The buildup of the two laser fields after the incoherent pumps have been switched on at $t=0$ to their constant values is shown in Fig. 6 for copropagating $\alpha$ and $\beta$ fields, $\Delta_{\alpha}^{c}=$ $-200 \mathrm{MHz}, \Delta_{\beta}^{c}=250 \mathrm{MHz}, \Delta \omega_{D \alpha}=333 \mathrm{MHz}$, and other parameters as specified above. Soon after population inversion at the drive transition appears, the $\beta$ field increases, showing relaxation oscillations which also manifest themselves in the population difference $n_{21}$ [see Figs. 6(a) and 6(b)]. When the $\beta$ field alone is close to a steady state the $\alpha$ field switches on in the absence of either one- or two-photon inversion [see Figs. 6(c) and 6(d)]. As $\alpha$-LWI is due to the predominance of one-photon gain processes, its switching on brings population into the lower level of the driven transition which results in a transient drecrease of the drive field. In the steady state, the switching on of the $\alpha$ field gives rise 

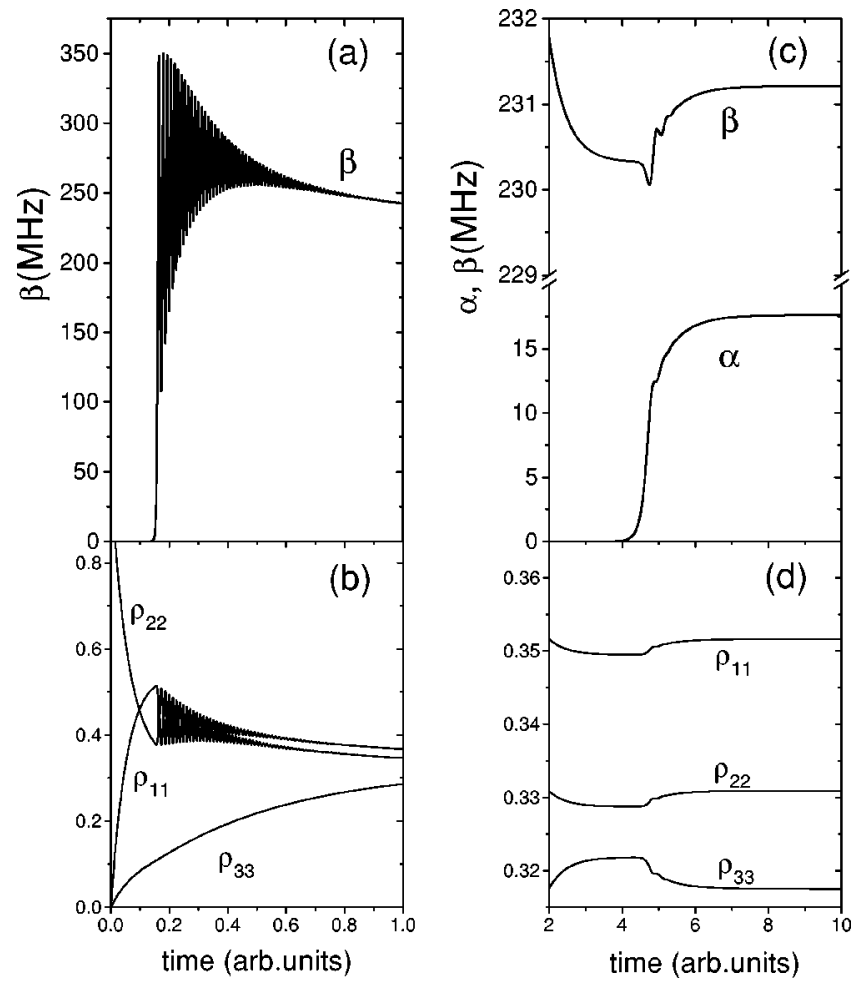

FIG. 6. The same as in Fig. 3 but now for the V scheme. The two laser beams are copropagating, $\Delta_{\alpha}^{c}=200 \mathrm{MHz}, \Delta_{\beta}^{c}=250 \mathrm{MHz}$, $\Delta \omega_{D \alpha}=333.02 \mathrm{MHz}$, and other parameters are given in the text.

through the pumping process $\Lambda^{\prime}$ to an increase of the population inversion in the driven transition and therefore to an increase of the $\beta$ intensity.

Figure 7 shows the amplitude of the generated $\alpha$ and $\beta$ fields as a function of Doppler broadening $\Delta \omega_{D \alpha}$ for cavity tunings $\Delta_{\alpha}^{c}=-200 \mathrm{MHz}$ and $\Delta_{\beta}^{c}=200 \mathrm{MHz}$. The resulting field detunigs at $\Delta \omega_{D \alpha}=0$ are $\Delta_{\alpha}=-213.14 \mathrm{MHz}$ and $\Delta_{\beta}$ $=97.41 \mathrm{MHz}$, which correspond to point $P$ in the inset. If these were the nominal detunings for all $\Delta \omega_{D \alpha}$, the points representing different atomic velocity classes in the inset would lie on the straight line with slope $R=1.88$ whose length depends on $\Delta \omega_{D \alpha}$. Actually, dispersive effects are different for different $\Delta \omega_{D \alpha}$ values and this means that not only the length of the line but also point $P$ must be changed in each case. Since only atoms corresponding to points in the segment $\overline{Q R}$ contribute to gain, one understands that $\alpha$ decreases and eventually disappears when $\Delta \omega_{D \alpha}$ increases. It is interesting to remark that the $\alpha$-lasing domain extends in Fig. 7 to values of $\Delta \omega_{D \alpha} \sim 1 \mathrm{GHz}$, which are typical values of the Doppler broadening at optical frequencies for a vapor cell.

\section{PRACTICAL CONSIDERATIONS}

Although the study presented in this paper is intended to give a general insight into a novel dual-wavelength laser that operates with population inversion at one of the wavelengths and without inversion at the other, we give below some considerations regarding experimental systems in which this novel laser could be realized.

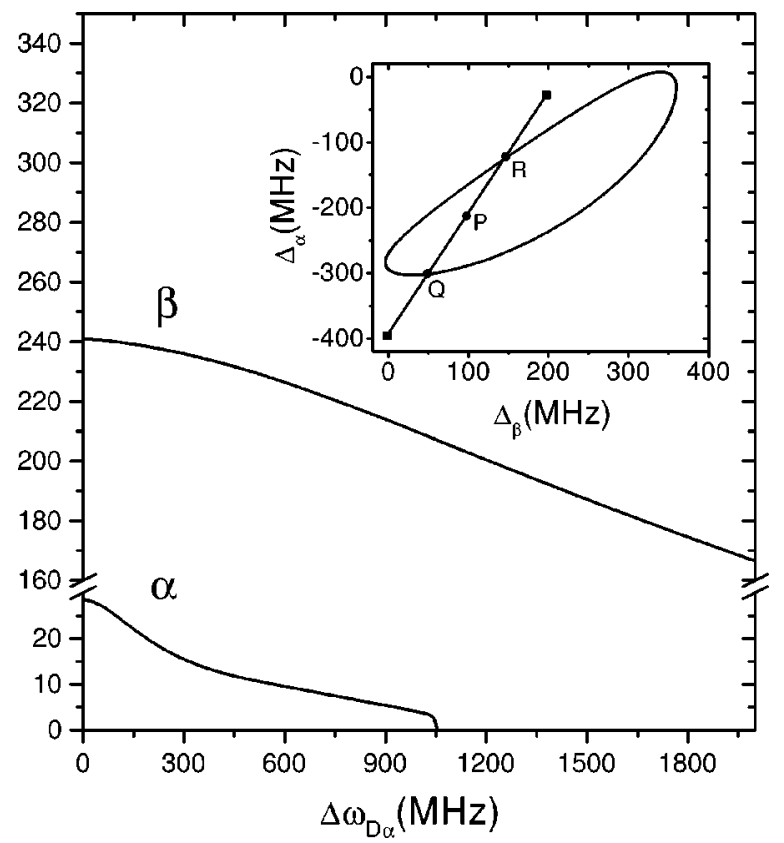

FIG. 7. The same as in Fig. 4 but now for the V scheme. The two laser beams are copropagating, $\Delta_{\alpha}^{c}=-200 \mathrm{MHz}, \Delta_{\beta}^{c}=200$ $\mathrm{MHz}$, and other parameters are given in the text. The line in the inset corresponds to $\Delta \omega_{D \alpha}=144.5 \mathrm{MHz}$.

In Sec. II A we considered a cascade-type system in atomic barium that is a nearly ideal closed three-level system, as discussed in [10]. Our model assumed for simplicity that an unidirectional incoherent pump mechanism $\Lambda^{\prime}(\Lambda)$ inverts the population at the transition $|2\rangle-|1\rangle$ (excites a small population to the upper level $|3\rangle$ ). In practice, however, the pump mechanisms $\Lambda^{\prime}$ and $\Lambda$ must involve additional levels to which the atoms are first excited an then decay radiatively or nonradiatively to levels $|2\rangle$ and $|3\rangle$, respectively. Our model applies strictly only when these decays are so fast that the population of the extra levels can be neglected. The implementation of incoherent pump schemes in barium would not be an easy task because there are no additional levels close to levels $|2\rangle$ and $|3\rangle$. From this perspective a cascade in neon atoms is much more convenient [see Fig. 8(a)]. The upper level $3 s_{2}$ can be easily excited in the usual way with an electrical discharge in a He-Ne mixture that produces a population inversion between levels $3 s_{2}$ and $2 p_{4}$. The generated drive field $(\beta)$ would be in this case the popular red line at $\lambda_{\beta}=632.8 \mathrm{~nm}$. Since the lifetime of the $1 s_{4}$ level is longer that of level $2 p_{4}$, the population in this transition cannot be inverted and the $\alpha$ field at $\lambda_{\alpha}$ $=609.6 \mathrm{~nm}$ could be generated without inversion. The frequency upconversion ratio for this system is $R=1.04$, which is very convenient for avoiding problems with Doppler broadening [8]. Moreover, this system would convert electrical input energy into laser output energy. In case the difference in lifetimes of levels $2 p_{4}$ and $1 s_{4}$ is not large enough to assure noninversion at this transition, one could replace $1 s_{4}$ by $1 s_{5}(J=2)$. This level cannot decay radiatively to the ground state ${ }^{1} S_{0}$. It decays via collisional relaxation and this can be controlled experimentally. Using $1 s_{5}$ as the lower $\alpha$ 
(a)
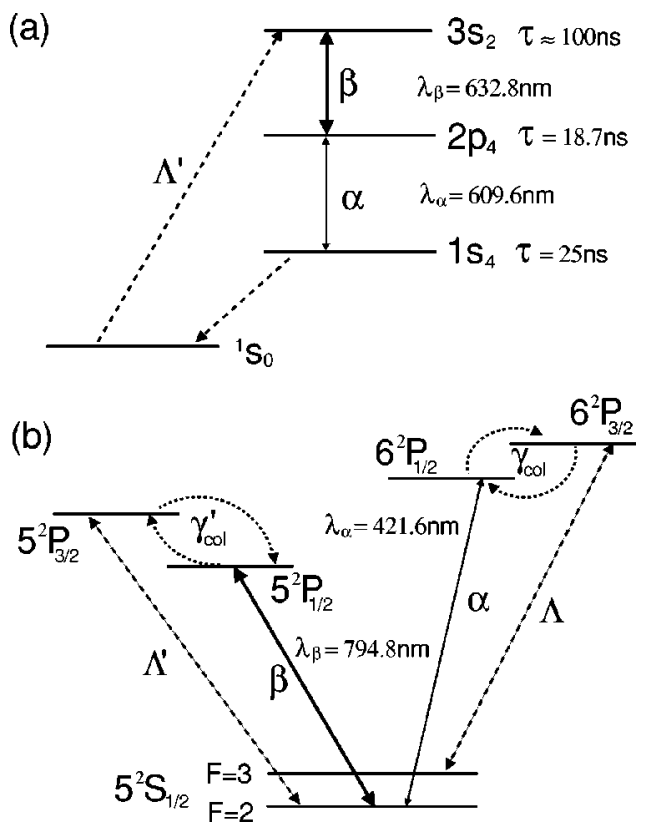

FIG. 8. (a) Simplified level scheme of neon. (b) Simplified level scheme of rubidium. Dotted arrows indicate collisional finestructure mixing of the rubidium excited levels. Other symbols have the same meaning as in Fig. 1.

-laser level would represent $\lambda_{\alpha}=594.5 \mathrm{~nm}$, i.e., $R=1.06$.

As for the V-type scheme in ${ }^{85} \mathrm{Rb}$, the incoherent optical pumping schemes needed to invert the population at the driven transition and to pump some population to the upper $\alpha$-laser level could be implemented in the following way, inspired in the nice papers by Kleinfeld and Streater $[14,15]$ reporting experiments on potassium-rare-gas mixtures. The relevant ${ }^{85} \mathrm{Rb}$ energy levels are shown in Fig. 8(b) where, for simplicity, magnetic sublevels and upper-level hyperfine structure are ignored. In this scheme, the drive field could be generated from the transition $5^{2} S_{1 / 2}(F=2)-5^{2} P_{1 / 2}$ at $\lambda_{\beta}$ $=794.8 \mathrm{~nm}$ if there is a large enough population inversion. This can be accomplished by optical excitation of the $5^{2} S_{1 / 2}(F=2)-5^{2} P_{3 / 2}$ transition in a rubidium-noble-gas mixture. Fine structure mixing of the rubidium excited levels provides an incoherent population mechanism of the $5^{2} P_{1 / 2}$ $[14,15]$ while the lower level of the drive transition is depleted by optical pumping to the $F=3$ hyperfine level. In order to eliminate any coherent coupling between the drive field to be generated and the optical field producing the excitation of the rubidium-noble-gas mixture, this could be realized by means of a noisy diode laser. The inversionless $\alpha$-laser field would be generated from transition $5^{2} S_{1 / 2}(F$ =2) $-6^{2} P_{1 / 2}$ at $\lambda_{\alpha}=421.6 \mathrm{~nm}$. Incoherent population of the $6^{2} P_{1 / 2}$ level can be obtained by means of (weak) optical excitation of transition $5^{2} S_{1 / 2}(F=3)-6^{2} P_{3 / 2}$ followed by fine-structure mixing of the $6 P$ levels via buffer gas collisions.

\section{CONCLUSIONS}

We have studied a model for an incoherently pumped laser operating with a Doppler-broadened three-level system placed in a doubly resonant cavity and producing two laser fields, one of them without population inversion. Each laser field couples to only one of the transitions of the three-level medium. We have studied our model for parameters appropiate to two real atomic systems: (i) a cascade scheme in atomic ${ }^{138} \mathrm{Ba}$ looking for lasing with (without) inversion at $\lambda_{\beta}=554 \mathrm{~nm}\left(\lambda_{\alpha}=821 \mathrm{~nm}\right)$ and (ii) a $\mathrm{V}$ scheme in atomic ${ }^{85} \mathrm{Rb}$ with ordinary lasing at $\lambda_{\beta}=780 \mathrm{~nm}$ and LWI at $\lambda_{\alpha}$ $=421.6 \mathrm{~nm}$. These two cases are qualitatively different in that two-photon processes are responsible for inversionless gain in case (i) while LWI in case (ii) arises from one-photon processes. Moreover, the ratio $R=\omega_{\alpha} / \omega_{\beta}$ of inversionless laser frequency $\omega_{\alpha}$ to ordinary laser frequency $\omega_{\beta}$ is $R$ $=0.67$ for the case of ${ }^{138} \mathrm{Ba}$ and it is $R=1.88$ for the case of ${ }^{85} \mathrm{Rb}$. In spite of the fact that the frequencies of the two laser fields are relatively different, our results show that dualwavelength lasing extends up to Doppler-broadening values $(\sim 1 \mathrm{GHz})$ typical for optical transitions of atoms in a vapor cell. In case (i) $\alpha$ lasing occurs without two-photon inversion only for approximately the lower half of the domain of Doppler-broadening values. However, in case (ii) one has LWI in all the domain. Some considerations for a practical realization of the dual-wavelength inversionless conventional laser have been discussed.

Finally, let us mention that we restricted ourselves here to study mainly the $\mathrm{cw}$ regime of our dual-wavelength laser. However, this system could exhibit interesting dynamic behavior due to the nonlinear coupling between the two laser fields.

\section{ACKNOWLEDGMENTS}

It is a pleasure to thank Ennio Arimondo for fruitful discussions on the present topic. We acknowledge support from the DGESIC (Spanish Government) under Contract Nos. PB95-0778-C02-02 and PB98-0935-C03-03. V.A. acknowledges support from the CIRIT (Catalan Government) for a grant (No. 1998FI 00194PG).
[1] For reviews see E. Arimondo, Coherent Population Trapping in Laser Spectroscopy, Progress in Optics Vol. XXXV, edited by E. Wolf (Elsevier Science, Amsterdam, 1996) p. 257; M. O. Scully and M. S. Zubairy, Quantum Optics (Cambridge University Press, Cambridge, England, 1997), Chaps. 7 and 14. See also H. Lee, M. Fleischhauer, and M. O. Scully, Phys. Rev. A 58, 2587 (1998) and references therein.

[2] See, e.g., O. Kocharovskaya, Phys. Rep. 219, 175 (1992); M. O. Scully, ibid. 219, 191 (1992); P. Mandel, Contemp. Phys.
34, 235 (1993); O. Kocharovskaya, Laser Phys. 5, 284 (1995).

[3] O. Svelto, Principles of Lasers, 3rd ed. (Plenum Press, New York, 1989), p. 371.

[4] A. S. Zibrov, M. D. Lukin, D. E. Nikonov, L. Hollberg, M. O. Scully, V. L. Velichansky, and H. G. Robinson, Phys. Rev. Lett. 75, 1499 (1995).

[5] G. G. Padmabandu, G. R. Welch, I. N. Shubin, E. S. Fry, D. E. Nikonov, M. D. Lukin, and M. O. Scully, Phys. Rev. Lett. 76, 2053 (1996). 
[6] F. B. de Jong, A. Mavromanolakis, R. J. C. Spreeuw, and H. B. van Linden van den Heuvell, Phys. Rev. A 57, 4869 (1998).

[7] M. D. Lukin, M. O. Scully, G. R. Welch, E. S. Fry, L. Hollberg, G. G. Padmabandu, H. G. Robinson, and A. S. Zibrov, Laser Phys. 6, 436 (1996).

[8] V. Ahufinger, J. Mompart, and R. Corbalán, Phys. Rev. A 60, 614 (1999).

[9] All frequencies given in this paper are true (not angular) frequencies.

[10] P. B. Sellin, G. A. Wilson, K. K. Meduri, and T. W. Mossberg,
Phys. Rev. A 54, 2402 (1996).

[11] J. Mompart and R. Corbalán, Opt. Commun. 156, 133 (1998).

[12] J. Mompart, V. Ahufinger, F. Silva, R. Corbalán, and R. Vilaseca, Laser Phys. 9, 844 (1999).

[13] S. F. Yelin, M. D. Lukin, M. O. Scully, and P. Mandel, Phys. Rev. A 57, 3858 (1998).

[14] J. A. Kleinfeld and A. D. Streater, Phys. Rev. A 49, R4301 (1994).

[15] J. A. Kleinfeld and A. D. Streater, Phys. Rev. A 53, 1839 (1996). 